\title{
Commentaire
}

\section{Changements climatiques, santé et avantages connexes des espaces verts}

\author{
Marianne Kingsley, M. Sc. (1); EcoHealth Ontario (2)
}

Diffuser cet article sur Twitter

\begin{abstract}
Résumé
Nous avons examiné deux des défis actuels de l'humanité, soit les changements climatiques et les maladies chroniques, en fonction des avantages connexes que les espaces verts procurent à la santé humaine et à l'environnement. La réduction de plusieurs maladies chroniques et des symptômes qui les accompagnent, dont l'anxiété, l'obésité et les maladies cardiovasculaires, a été associée à la présence d'espaces verts et à l'accès à ceux-ci. Les espaces verts offrent également à un certain nombre d'avantages pour la santé environnementale : ils réduisent le risque d'inondation, améliorent la qualité de l'air, rafraîchissent la température et créent de l'ombre. Ces avantages touchent à la fois les symptômes de plusieurs maladies chroniques ainsi que les facteurs de risque associés et les effets des changements climatiques sur l'environnement et la santé. Notre article porte sur les façons d'optimiser les avantages connexes des espaces verts, au moyen de deux exemples de collaborations multisectorielles. À l'aide de ces deux exemples, nous avons conçu un modèle de collaboration collective visant à régler simultanément des problèmes complexes, comme les changements climatiques et les maladies chroniques, grâce à l'intervention sur les espaces verts.
\end{abstract}

Mots-clés : maladie chronique, espace vert, changement climatique, écosanté, santé publique, avantages connexes.

\section{Introduction}

À l'échelle locale comme à l'échelle mondiale, l'humanité est confrontée à deux problèmes de fond qui ont une incidence sur la santé humaine : les maladies chroniques $^{1}$ et les changements climatiques ${ }^{2}$. Les principales maladies chroniques, dont le cancer, les maladies cardiaques et le diabète, sont responsables chaque année de $65 \%$ de tous les décès au Canada ${ }^{1}$. Les impacts des changements climatiques, en particulier l'augmentation de phénomènes météorologiques extrêmes comme des épisodes de chaleur extrême, des périodes de sécheresse, des feux de forêt et des inondations, se font sentir sur l'ensemble du territoire ${ }^{2}$. Les effets indirects des changements climatiques se révèlent aussi de plus en plus importants, surtout si on tient compte de l'aggravation des problèmes de santé publique et de la menace qui pèse sur les gains en santé des populations ${ }^{3,4}$. Bien que ces effets touchent l'ensemble de la population, les groupes vulnérables sont affectés de façon disproportionnée ${ }^{5}$.

Les espaces verts (encadré 1) constituent une intervention unique qui offre des avantages connexes sur le plan de l'atténuation des effets des changements climatiques, de l'adaptation à ces effets et de la santé humaine. La présence d'espaces verts urbains et l'accès à ceux-ci réduisent le taux et l'incidence des maladies chroniques $^{6,7}$. Les espaces verts peuvent à la fois contribuer à atténuer les changements climatiques et leurs effets et améliorer la résilience à ces changements 8 .
Points saillants

- Les principales maladies chroniques, dont le cancer, les maladies cardiaques et le diabète, sont responsables chaque année de $65 \%$ des décès au Canada.

- Les effets des changements climatiques, en particulier l'augmentation des épisodes de chaleur extrême, des périodes de sécheresse et des inondations, se font sentir sur l'ensemble du territoire.

- La protection, l'amélioration et l'extension des espaces verts peuvent aider à régler ces deux grands problèmes.

- EcoHealth Ontario est un exemple de partenariat multisectoriel dans le cadre duquel les intervenants collaborent pour atteindre plusieurs objectifs en matière de santé publique, de planification et d'environnement.

Comme le résume l'encadré 2 , les espaces verts favorisent l'activité physique, contribuent à l'interaction et à la cohésion sociales, accroissent l'accès à une alimentation saine et favorisent la réduction du stress et le rétablissement des fonctions cognitives ${ }^{6-8}$. Ils améliorent également la qualité de l'air, créent de l'ombre, réduisent les températures de l'air extérieur et diminuent le risque d'inondation ${ }^{9-11}$

Cet article décrit les défis actuels en matière de santé et les menaces liées aux changements climatiques auxquels font face les Canadiens et explique comment les espaces verts, par leurs avantages connexes,

Rattachement des auteurs :

1. Healthy Public Policy, Toronto Public Health, Ville de Toronto, Toronto (Ontario) Canada

2. EcoHealth Ontario, Ontario, Canada

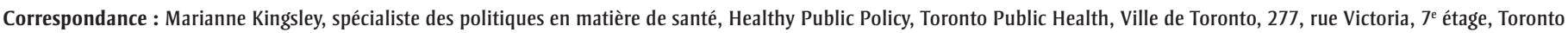
(Ontario) M5B 1W2; tél. : 416-338-5150; courriel : marianne.kingsley@toronto.ca 


\section{ENCADRÉ 1}

Le terme « espace vert » désigne un vaste éventail d'espaces

\section{Qu'est-ce qu'un espace vert?}

II existe de nombreux types d'espaces verts, tous pouvant offrir des bénéfices en matière de santé, d'atténuation des effets des changements climatiques et d'adaptation à ces effets. Le terme " espace vert » désigne les infrastructures vertes, les espaces naturels, les espaces ouverts et les espaces verts aménagés. Ces espaces sont de divers types, tailles et fonctions et peuvent constituer :

- des espaces publics, comme les parcs, les aires de conservation, les couloirs de verdure, les sentiers, les forêts urbaines et rurales, les arbres de rue, les jardins communautaires, les terrains scolaires, les littoraux et les versants de vallées ;

- des espaces et infrastructures privés et institutionnels comme les jardins, les toits verts, les murs verts, les cimetières, les terrains de golf et autres espaces extérieurs.

offrent une occasion irremplaçable d'atténuer les effets de ces changements climatiques. Il présente également deux exemples de collaboration, EcoHealth Ontario au Canada et Climate Change Parks en Écosse (Royaume-Uni), qui visent à s'attaquer à plusieurs enjeux transversaux, en l'occurrence les changements climatiques, la santé publique et l'environnement, en encourageant le recours aux espaces verts en tant qu'intervention efficace en matière de lutte contre les changements climatiques.

\section{Maladies chroniques et espaces verts}

On compte quatre grandes maladies chroniques, et environ $20 \%$ des Canadiens vivent avec au moins l'une d'entre elles ${ }^{1}$. Le cancer, les maladies cardiovasculaires, le diabète et les maladies respiratoires sont responsables d'environ $65 \%$ de tous les décès au Canada ${ }^{1}$. De plus, les troubles de santé mentale, dont la dépression et l'anxiété, sont la principale cause d'invalidité au travail au Canada ${ }^{1}$. Qui plus est, un tiers des dépenses directes en soins de santé au Canada sont attribuables aux troubles des appareils circulatoire et respiratoire, aux troubles musculosquelettiques et aux troubles de santé mentale ${ }^{19}$. Plusieurs facteurs de risque sont associés au risque de maladie chronique d'une personne, notamment son niveau d'activité physique, son exposition à la fumée de tabac et ses habitudes alimentaires ${ }^{1}$. Par exemple, neuf enfants canadiens sur dix ne respectent pas le niveau d'activité physique recommandé ${ }^{1}$. Toutefois, ces facteurs de risque sont modifiables, et la présence d'espaces verts et l'accès à ceux-ci peuvent réduire certains facteurs de risque menant à des maladies chroniques $^{7}$

Il existe un vaste corpus de données probantes à propos des effets positifs sur la santé de la présence d'espaces verts et de l'accès à ceux-ci ${ }^{7}$ D'après ces études, les bienfaits des espaces verts pour la santé se concrétisent de diverses façons (encadré 2). Les espaces verts ont plusieurs effets positifs sur l'environnement urbain favorisant la santé des populations, en particulier la réduction du bruit, la création d'ombre et de fraîcheur et la diminution des risques d'inondation et de pollution atmosphéri$q^{9} e^{9-11}$. De plus, ils peuvent favoriser la santé et le bien-être en permettant la réduction du stress et la relaxation, l'activité physique, l'amélioration des interactions sociales et la cohésion sociale $e^{6-8}$. Les principaux bienfaits pour la santé associés à la présence d'espaces verts et à l'accès à ceux-ci sont de meilleurs niveaux en santé mentale, une meilleure condition physique, de meilleures fonctions cognitives et immunitaires ainsi que des taux de mortalité inférieurs ${ }^{12,13}$.

Les bienfaits des espaces verts sur la santé sont particulièrement importants chez ceux qui subissent des inégalités en matière de santé $^{16}$. Par exemple, une étude menée en Angleterre a révélé que la mortalité toutes causes confondues, la mortalité due aux maladies circulatoires et les inégalités de revenu entre le groupe de défavorisation minimale et le groupe de défavorisation maximale étaient nettement inférieures chez ceux vivant dans un quartier où le nombre d'espaces verts était plus élevé ${ }^{16}$.

Une constatation similaire est ressortie d'une étude menée à Toronto et consacrée à la densité des arbres en lien avec les états de santé et les maladies cardiométaboliques autodéclarés : la présence d'arbres améliorait considérablement la perception de la santé, la rapprochant de celle associée à un quartier où le revenu médian était supérieur de 10000 dollars ou au fait d'avoir 7 ans de moins ${ }^{20}$.

\section{Les espaces verts peuvent améliorer la résilience aux changements climatiques}

Comme l'illustrent les encadrés 2 et 3, la présence d'espaces verts aide à atténuer les changements climatiques et à améliorer la santé humaine en réduisant les facteurs de risque de maladies chroniques. Les espaces verts offrent également les avantages connexes d'améliorer la résilience aux changements climatiques et le rétablissement à la suite de l'exposition aux effets des changements climatiques (encadré 3) 21,22.

Les inondations illustrent la façon dont la présence d'espaces verts et l'accès à ceuxci procurent des avantages connexes. Alors que les inondations sont à la hausse en lien avec les changements climatiques, la présence d'espaces verts peut en réduire le risque de dommages et l'étendue. En effet, les espaces verts (parcs, rigoles biologiques, jardins pluviaux, milieux humides aménagés et prés en plaine inondable) peuvent stocker temporairement les eaux pluviales et réduire le ruissellement ${ }^{2}$. De plus, et parallèlement, la présence d'espaces verts et l'accès à ceux-ci peuvent contribuer à réduire les symptômes associés à la santé mentale, tels que le stress et l'anxiétée, qui peuvent être exacerbés par une inondation $^{23}$. Selon le Centre Intact d'adaptation au climat, trois ans après l'inondation de leur maison, $48 \%$ des répondants des ménages ayant subi une inondation étaient inquiets en cas de pluie, contre $3 \%$ des répondants des ménages n'en ayant pas subi $^{23}$.

\section{Mettre en pratique les données probantes sur les espaces verts grâce à des collaborations multisectorielles}

La protection, la promotion et l'augmentation des espaces verts peuvent être bénéfiques pour la santé des populations grâce à : 1) la réduction de certaines maladies chroniques et des facteurs de risque connexes, 2) l'atténuation des effets des changements climatiques et 3) l'augmentation de la résilience à la suite d'une exposition aux effets des changements climatiques $^{9-11}$. Ces enjeux relèvent de nombreux secteurs, allant des responsables de la santé publique aux urbanistes en passant par les défenseurs de l'environnement. 
ENCADRÉ 2

Avantages connexes des espaces verts et impact sur les changements climatiques et la santé humaine

Impact des espaces verts sur la santé et sur l'environnement

La présence d'espaces verts et l'accès à ceux-ci sont associés de façon positive à des facteurs liés à l'environnement et à la santé humaine ${ }^{6}$.

Associations entre espaces verts et environnement ${ }^{9-11}$

Associations entre espaces verts et santé et bien-être des populations $^{6-8}$

- Amélioration de la qualité de l'air

- Réduction des îlots de chaleur urbains, création d'ombre

- Atténuation des inondations grâce au stockage des eaux pluviales

- Réduction du bruit

- Approvisionnement en aliments
L'accès à des espaces verts dans un quartier est corrélé à :

- Une amélioration des résultats à la naissance

- Une réduction de la mortalité, toutes causes confondues

- Une réduction du niveau d'obésité

- Une réduction du nombre de personnes atteintes de maladies cardiovasculaires

- Une amélioration des symptômes de maladie mentale, dont la dépression et l'anxiété

- Une diminution des sentiments de stress autodéclarés

- Une amélioration de la cohésion sociale

Facteurs influant sur les avantages des espaces verts

Caractéristiques des espaces verts

- Disponibilité et accessibilité (emplacement, distance du domicile, nombre, taille ${ }^{12,13}$ )

- Esthétique (aménagement paysager, perception de la qualité ${ }^{13}$ )

- Équipements (infrastructures, services ${ }^{14}$ )

- Entretien (régularité de l'entretien, enlèvement des ordures ${ }^{15}$ )

Les espaces verts qui ont les plus grandes répercussions sur la santé sont ceux situés à proximité des résidences, accessibles et utilisables par divers groupes et perçus comme bien entretenus ${ }^{7}$.

\section{Populations}

On a constaté que ce sont les populations vulnérables qui profitent le plus des espaces verts, en particulier les personnes à faible revenu, les groupes racialisés, les personnes âgées et les enfants ${ }^{16}$. En particulier, la présence de parcs bien entretenus avec des terrains de jeu à proximité de leur domicile a une incidence positive sur la santé des enfants ${ }^{17}$. Les espaces verts ont une incidence favorable sur la santé des groupes vulnérables, et ce, même si l'augmentation de la densité de ces espaces dans le voisinage n'est que relativement faible ${ }^{18}$.

ENCADRÉ 3

Effets des changements climatiques sur la santé humaine et avantages connexes des espaces verts

\section{Effets des changements climatiques sur la santé}

Maladies et décès prématurés attribuables à l'exposition à la chaleur extrême $e^{2,5}$

Maladies, stress et décès prématurés attribuables à l'exposition aux inondations $\mathrm{s}^{2,10}$

Stress psychologique causé par les effets des conditions météorologiques extrêmes ${ }^{2}$

Insécurité alimentaire ${ }^{5}$

Maladies cardiovasculaires et respiratoires attribuables à la détérioration de la qualité de l'air ${ }^{3}$

\section{Avantages connexes des espaces verts ou atténuation}

- Création d'ombre ${ }^{11}$

- Réduction de l'effet d'îlot de chaleur ${ }^{11}$

- Réduction du risque d'inondation par la diminution du ruissellement ${ }^{21}$

- Réduction du stress, de l'anxiété et de la dépression

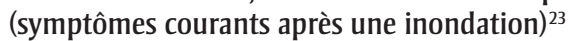

- Accès à une source alimentaire locale grâce aux jardins communautaires $^{7}$

- Amélioration de la qualité de l'air ${ }^{9}$

- Réduction du taux de maladies cardiovasculaires ${ }^{8}$ 
Ainsi, divers secteurs peuvent se réunir, collaborer et agir davantage collectivement autour de la protection, de la promotion et de l'augmentation des espaces verts. Ils peuvent se réunir et collaborer dans le cadre d'une intervention commune et optimiser ainsi les avantages connexes que procurent les espaces verts. EcoHealth Ontario au Canada et Climate Change Parks [Parcs pour contrer les changements climatiques] en Écosse offrent deux exemples de collaboration fructueuse pour la promotion des espaces verts.

EcoHealth Ontario est un groupe multisectoriel collaboratif qui tire parti d'activités de renforcement mutuel et qui considère que la protection, la promotion et l'augmentation des espaces verts sont bénéfiques pour la santé des populations. Cette collaboration a donné lieu à plusieurs ateliers multisectoriels dans le cadre desquels des planificateurs, des responsables de la santé publique et des écologistes, entre autres intervenants, discutant des méthodes, des outils et des stratégies susceptibles d'aider les spécialistes à mettre en œuvre les interventions liées aux espaces verts. Elle permet également de produire des rapports, des trousses à outils et du matériel éducatif destinés à promouvoir les bienfaits des espaces verts sur l'environnement, la santé et le bien-être, en insistant sur l'atténuation des effets des changements climatiques, l'adaptation à ces effets et la réduction des maladies chroniques.

L'aménagement novateur de Climate Change Parks en Écosse relève aussi d'une collaboration multisectorielle axée sur les avantages connexes des espaces verts. Cette initiative vise à rénover les espaces verts urbains déjà en place pour fournir des solutions devant les changements climatiques $^{24}$. Elle consiste à définir la façon dont les divers éléments d'un parc peuvent être modifiés de manière à avoir une faible empreinte carbone, à s'adapter aux effets des changements climatiques (par exemple en assurant la gestion des inondations et en créant de l'ombre) et à rendre les espaces verts agréables dans diverses situations ${ }^{24}$.

\section{Conclusion}

Protéger, promouvoir, augmenter et améliorer les espaces verts procure plusieurs avantages connexes touchant certains des grands enjeux auxquels font face les collectivités aujourd'hui. Le fait de mettre l'accent sur les espaces verts offre une occasion irremplaçable de créer une intervention unique comportant de multiples avantages pour de multiples intervenants. Reconnaître les bénéfices qu'ils procurent permet de protéger et de promouvoir ces espaces, particulièrement dans les centresvilles.

Il est impératif que gouvernements, associations, organismes et entreprises unissent leurs efforts pour atteindre un objectif commun, surtout face à ces défis complexes. La collaboration multisectorielle constitue un moyen précieux d'optimiser les avantages connexes que procurent les espaces verts. Collaborer pour faire des espaces verts une priorité pour les collectivités et à l'échelle locale va contribuer à résorber les problèmes associés aux maladies chroniques et aux changements climatiques.

\section{Conflits d'intérêts}

Les auteurs déclarent n’avoir aucun conflit d'intérêts.

\section{Contributions des auteurs et avis}

MK a contribué à la conception de l'étude ainsi qu'à la rédaction du manuscrit. Les membres d'EcoHealth Ontario ont pris part à la conception de l'étude, à la révision critique du manuscrit et à l'approbation de la version soumise pour évaluation.

Le contenu de l'article et les points de vue qui y sont exprimés n'engagent que les auteurs; ils ne correspondent pas nécessairement à ceux du gouvernement du Canada.

\section{Références}

1. Agence de la santé publique du Canada. Quel est l'état de santé des Canadiens? [Internet]. Ottawa (Ont.) : Agence de la santé publique du Canada; 2016. En ligne à : https://www .canada.ca/fr/sante-publique/services /publications/vie-saine/quel-est-l-etat -sante-des-canadiens.html

2. Gouvernement du Canada. Vivre avec les changements climatiques au Canada : perspectives des secteurs relatives aux impacts et à l'adaptation [Internet]. Ottawa (Ont.) : Ressources naturelles Canada; 2014. En ligne à : https://www .rncan.gc.ca/sites/www.nrcan.gc.ca /files/earthsciences/pdf/assess/2014 /pdf/Rapport-complet_Fra.pdf
3. Watts N, Amann M, Arnell N, et al. The 2018 report of the Lancet Countdown on health and climate change: shaping the health of nations for centuries to come. The Lancet. 2018;392(10163):2479-2514.

4. Watts N, Adger W, Agnolucci P, et al. Health and climate change: policy responses to protect public health. The Lancet. 2015;386(10006):1861-1914.

5. Intergovernmental Panel on Climate Change (IPCC). Global warming of $1.5^{\circ} \mathrm{C}$. An IPCC Special Report on the impacts of global warming of $1.5^{\circ} \mathrm{C}$ above pre-industrial levels and related global greenhouse gas emission pathways, in the context of strengthening the global response to the threat of climate change, sustainable development, and efforts to eradicate poverty [Internet]. Genève (Suisse) : World Meteorological Organization; 2018. En ligne à : http://www.ipcc.ch/report /sr15/

6. James P, Banay R, Hart J, Laden F. A review of the health benefits of greenness. Current Epidemiology Reports. 2015;2(2):131-142.

7. Toronto Public Health. Green City: why nature matters to health - an evidence review [Internet]. Toronto: Toronto Public Health; 2015. En ligne à : https://www.toronto.ca/legdocs /mmis/2015/hl/bgrd/backgroundfile -83421.pdf

8. Sandifer P, Sutton-Grier A, Ward B. Exploring connections among nature, biodiversity, ecosystem services, and human health and well-being: opportunities to enhance health and biodiversity conservation. Ecosystem Services. 2015;12:1-15.

9. Nowak D, Hirabayashi S, Bodine A, Greenfield E. Tree and forest effects on air quality and human health in the United States. Environ Pollut. 2014; 193:119-129.

10. Depietri Y, Renaud F, Kallis G. Heat waves and floods in urban areas: a policy-oriented review of ecosystem services. Sustainability Science. 2011; 7(1):95-107. 
11. Bowler D, Buyung-Ali L, Knight T, Pullin A. Urban greening to cool towns and cities: a systematic review of the empirical evidence. Landsc Urban Plan. 2010;97(3):147-155.

12. Maas J, Verheij R, de Vries S, Spreeuwenberg P, Schellevis F, Groenewegen P. Morbidity is related to a green living environment. J Epidemiol Community Health. 2009;63(12):967973.

13. de Vries S, van Dillen S, Groenewegen P, Spreeuwenberg P. Streetscape greenery and health: stress, social cohesion and physical activity as mediators. Soc Sci Med. 2013;94:26-33.

14. Anthamatten P, Brink L, Lampe S, Greenwood E, Kingston B, Nigg C. An assessment of schoolyard renovation strategies to encourage children's physical activity. Int $\mathrm{J}$ Behav Nutr Phys Act. 2011;8(1):27.

15. Maas J, Verheij R, Spreeuwenberg P, Groenewegen P. Physical activity as a possible mechanism behind the relationship between green space and health: a multilevel analysis. BMC Public Health. 2008;8(1):206.

16. Mitchell R, Popham F. Effect of exposure to natural environment on health inequalities: an observational population study. The Lancet. 2008; 372(9650):1655-1660.

17. Potwarka L, Kaczynski A, Flack A. Places to play: association of park space and facilities with healthy weight status among children. J Community Health. 2008;33(5):344-350.

18. Mitchell R, Richardson E, Shortt N, Pearce J. Neighborhood environments and socioeconomic inequalities in mental well-being. Am J Prev Med. 2015;49(1):80-84.

19. Agence de la santé publique du Canada. Fardeau économique de la maladie au Canada, 2010 [Internet]. Ottawa (Ont.) : Agence de la santé publique du Canada; 2017. En ligne à : https://www.canada.ca/content /dam/phac-aspc/documents/services /publications/science-research/economic -burden-illness-canada-2010/fardeau -economique-maladie-canada-2010.pdf
20. Kardan O, Gozdyra P, Misic B, et al. Neighborhood greenspace and health in a large urban center. Sci Rep. 2015; 5(1):11610. doi: 10.1038/srep11610.

21. European Environment Agency. Urban adaptation to climate change in Europe: challenges and opportunities for cities together with supportive national and European policies [Internet]. Copenhagen (Danemark) : Office for Official Publications of the European Union; 2012. En ligne à : https://www .eea.europa.eu/publications/urban -adaptation-to-climate-change

22. World Health Organization (WHO). Urban green spaces and health: a review of evidence [Internet]. Copenhagen (Danemark) : WHO Regional Office for Europe; 2016. En ligne à : http:// www.euro.who.int/__data/assets /pdf_file/0005/321971/Urban-green -spaces-and-health-review-evidence .pdf?ua $=1$

23. Decent D, Feltmate B. Après les inondations : les effets du changement climatique sur la santé mentale et la perte de temps de travail [Internet]. Waterloo (Ont.) : Centre Intact d'adaptation au climat; 2018. En ligne à : https://www.centreintactadaptation climat.ca/wp-content/uploads//2018 /06/after_the_flood_report_FR.pdf

24. greenspace scotland. Retrofitting urban parks to deliver climate change actions [Internet]. Stirling (Scotland) : greenspace scotland; 2012. En ligne à : https://drive.google.com/file/d /1rWuEtaHSF21rUOxW9hVzAgRVUnq -ZdY7/view 